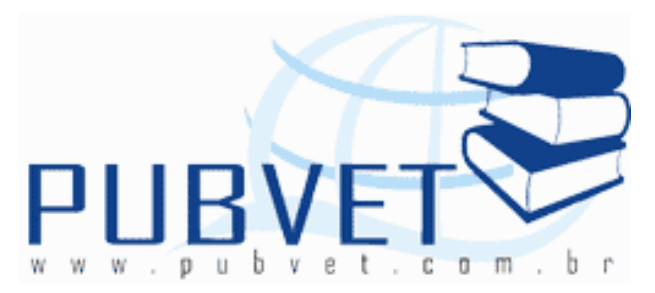

PUBVET, Publicações em Medicina Veterinária e Zootecnia.

\title{
Uso de gracilaria no desempenho de frango de corte
}

Bárbara Monique de Freitas Vasconcelos ${ }^{1}$ e Alex Martins Varela de Arruda $^{2}$

${ }^{1}$ Mestrando em Ciência Animal - Universidade Federal Rural do Semi-Árido (UFERSA) - Programa de Pós-Graduação em Ciência Animal (PPGCA) - Av. Francisco Mota, 572 - Bairro Costa e Silva - 59625-900 - Mossoró - RN. barbara.biotec1@gmail.com

${ }^{2}$ Professor Nutrição Avícola - UFERSA - PPGCA

\section{Resumo}

A avicultura brasileira vem se destacando dentro da economia nacional e atualmente é o setor mais produtivo da produção animal. As pesquisas relacionadas ao melhoramento do desempenho dos frangos de corte tem se destacado dentro do setor, principalmente após a proibição dos antibióticos. Diversos alimentos não convencionais dentro da avicultura estão sendo avaliados como promotor de crescimento, inclusive as macroalgas. Estas tem ganhado espaço principalmente devido ao elevado potencial nutricional, com teores de fibras excelentes que podem ser aproveitadas com prebióticos. A Gracilaria birdiae é uma espécie bastante cultivada no Brasil, em especial no Nordeste brasileiro, sua composição nutricional desperta interessa e a aponta como um promotor de crescimento em potencial. Portanto, as macroalgas vem demonstrando que podem ser aplicadas para uma melhora no desempenho de frangos de corte.

Palavras-chave: macroalgas, frango, desempenho nutricional, prebióticos 
VASCONCELOS, B.M.F. e ARRUDA, A.M.V. Uso de gracilaria no desempenho de frango de corte. PUBVET, Londrina, V. 8, N. 20, Ed. 269, Art. 1797, Outubro, 2014.

\title{
Use of gracilaria in performance broiler
}

\begin{abstract}
The brazilian poultry industry has been highlighted in the national economy and is currently the most productive sector of animal production. There search related to improving the performance of broilers has excelled within the industry, especially after the ban on antibiotics. Several unconventional foods with in the poultry industry are being evaluated as growth promoter, including macroalgae. These have gained ground mainly due to the high nutritional potential with levels of fine fibers that can be enjoyed with prebiotics. The Gracilaria birdiae is largely cultivated in Brazil, especially in the brazilian northeast, its nutritional composition arouses interest and points as a promoter of growth potential. Therefore, macroalgae has demonstrated that can be applied to an improvement in performance of broilers.
\end{abstract}

Keywords: macroalgae, chicken, nutritional performance, prebiotics

\section{1-INTRODUÇÃO}

A avicultura brasileira vem se mostrando capaz de atender as grandes demandas de carne de frango do mercado nacional e mundial. Com um mercado cada vez mais promissor e aberto, salvo as barreiras sanitárias e subsídios agrícolas dos outros países, o Brasil vem se mostrando cada vez mais competitivo e eficiente (MACHADO et al., 2010).

A participação brasileira no agronegócio de carnes foi evidenciada a partir de atuais dificuldades tecnológicas enfrentadas pelos países europeus, tais como na sanidade do produto final e na produção, que devem servir como alerta para a definição de estratégias apropriadas para o Brasil (ZYBERSTAJN \& GRAÇA, 2002).

Com a proibição na utilização de antibióticos promotores de crescimento (APC) pelo mercado consumidor, principalmente Comunidade Europeia, têm-se exigido da indústria, bem como dos pesquisadores, a procura de alternativas 
VASCONCELOS, B.M.F. e ARRUDA, A.M.V. Uso de gracilaria no desempenho de frango de corte. PUBVET, Londrina, V. 8, N. 20, Ed. 269, Art. 1797, Outubro, 2014.

para estes promotores, pois sua retirada total pode trazer prejuízos para a indústria avícola brasileira (MACHADO et al., 2010).

Os frequentes aumentos nos preços dos grãos de cereais e suplementos proteicos vegetais utilizados na alimentação animal têm despertado grande interesse pelo aproveitamento de alimentos conhecidos como não convencionais na indústria de alimentação do Brasil. Dentre os produtos que podem substituir os suplementos proteicos convencionais usados na alimentação animal, destacam-se as macroalgas, microalgas, bactérias, fungos e leveduras (BUTOLO, 1996).

As macroalgas tem se destacado no cenário mundial, devido as características nutricionais de elevado interesse econômico, principalmente a divisão Rhodophyta que apresenta elevado teor proteico. O objetivo desta revisão é atualizar o leitor sobre o uso de macroalgas (Gracilaria) na alimentação de frangos de corte.

\section{2- ALGAS MARINHAS}

As algas ocorrem em todo o globo, e é a base da cadeia alimentar, servindo como fonte de nutrientes para uma grande variedade de organismos aquáticos. Elas podem ser classificadas em macroalgas e microalgas. As macroalgas são aquelas visualizadas a olho nu, as microalgas necessitam de microscópio para serem observadas (MARINHO-SORIANO et al., 2011; RAVEN et al., 1996).

As macroalgas marinhas são vegetais aquáticos sem vascularização no qual o corpo é representado por um talo, a maioria são bentônicas, ou seja, vivem aderidas a um substrato, são autotróficas. Elas podem ser divididas em três grandes grupos: algas verdes, algas vermelhas e algas pardas (McHUGH, 2003; MENDEZ, 2002).

As algas verdes (Divisão Chlorophyta) são o grupo mais diversificado Elas possuem xantofila, carotenos e clorofila $a$ e $b$ como pigmentos, sendo o último o mais importante. As algas verdes podem ser marinhas ou dulcícolas (ALGAS VERDES, 2012). 
VASCONCELOS, B.M.F. e ARRUDA, A.M.V. Uso de gracilaria no desempenho de frango de corte. PUBVET, Londrina, V. 8, N. 20, Ed. 269, Art. 1797, Outubro, 2014.

As algas vermelhas (Divisão Rhodophyta) possuem aproximadamente 6000 espécies divididas em várias classes sendo a Gelidiales e Gracilariales as mais importantes. Essas algas possuem a ficoeritrina como principal pigmento, além disso, são bastante utilizadas na indústria alimentícia e microbiológica (MAURICIO et al., 2011).

As algas pardas (Divisão Phaeophyta) são o grupo mais estudado. Elas são fontes ricas de iodo e ferro, além disso, são bastante utilizadas como alimento humano. Os gêneros Laminaria, Himantalia e Sargassum apresentam-se como os principais deste grupo. Algumas espécies deste grupo podem chegar a $70 \mathrm{~m}$ de comprimento, formando os conhecidos Kelps (algas gigantes) (RAVEN et al., 1996).

\section{1 - Composição nutricional}

As propriedades nutricionais de algas são poucas conhecidas, em relação às de plantas terrestres, mas vários trabalhos têm mostrado que elas são pobres em lipídios, mas rico em proteínas, polissacarídeos, minerais e vitaminas (DARCY-VRILLON, 1993; DAWCZYNSKI et al., 2007; MABEAU e FLEURENCE, 1993).

As algas apresentam um alto teor de polissacarídeos contribuindo para o elevado conteúdo de fibras dietéticas nas algas. O teor destas fibras varia de acordo com as espécies de algas analisadas, apresentando uma média de 30 $40 \%$ do peso seco (RUPÉREZ et al., 2010; TABARSA et al., 2012).

Santos et al. (2012) avaliaram a composição centesimal da alga Kappaphycus alvarezii e encontraram um teor de $44 \%$ do peso seco em fibras, demonstrando um alto potencial nutricional. Carneiro et al. (2012) avaliaram o potencial nutricional (fibras) de duas algas vermelhas (Hypnea musciformis e Solieria filiformis) coletadas no litoral do Ceará - RN - Brasil. O resultado obtido mostrou um elevado conteúdo de fibras, $54 \%$ para $H$. musciformis e $49 \%$ para S. filiformis.

O teor de lipídios em algas é baixo (1-5\%), entretanto apresenta um elevado teor de ácidos graxos insaturados (PUFA). Isto é de grande 
VASCONCELOS, B.M.F. e ARRUDA, A.M.V. Uso de gracilaria no desempenho de frango de corte. PUBVET, Londrina, V. 8, N. 20, Ed. 269, Art. 1797, Outubro, 2014.

importância na alimentação, pois os PUFA's são benéficos na alimentação, sendo alguns essenciais ao metabolismo, e somente adquiridos através dos alimentos (PATARRA, 2008).

A fração mineral de algumas algas corresponde a $36 \%$ do peso seco total. As algas pardas são conhecidas como uma fonte rica em iodo (mineral que atua no metabolismo de lipídios). As algas da família das Laminaria são a principal fonte e podem conter entre 1500 a 8000 ppm do peso seco total. Algumas algas são importantes fontes de cálcio, o conteúdo deste mineral pode chegar até 7\% do peso seco total (PATARRA, 2008).

A alga Lithothamnium calcareum vem sendo bastante aplicada como fonte de suplemento de cálcio em rações animais. O esqueleto dela é constituído de 95 - 99\% de minerais, principalmente carbonato de cálcio e de fósforo. Ela pode ser usada na correção e fertilização do solo, nutrição animal e humana e empregada na indústria (MELO, 2006; GOETZ, 2008; COSTA NETO et al., 2010).

O teor proteico das algas difere de acordo com a espécie. As algas pardas possuem um conteúdo baixo, em torno de $5-15 \%$ do peso seco, enquanto as algas verdes e vermelhas apresentam valores entre $10-30 \%$ do peso seco. Os ácidos, aspártico e glutâmico constituem a maior fração de aminoácidos para a maior parte das algas marinhas (PATARRA, 2008).

Pires et al. (2012) determinaram a composição química da alga Gracilaria domingensis, uma rodofícea e demonstrou que o teor proteico dela é de $17 \%$ do peso seco. Calado et al. (2012) compararam o potencial nutricional de duas rodofíceas (Gracilaria cornea e Gracilaria domingensis) e obtiveram como teor proteico 16 e $17 \%$ do peso seco, respectivamente.

Estudos realizados por Frikha et al. (2011) com quatro espécies de macroalgas diferentes evidenciam as diferenças nutricionais das três divisões: Ulva rígida, Codium bursa (verdes), Ceramium diaphanum (vermelha) e Cystoseira barbata (parda). A alga parda Cystoseira barbata apresentou o maior nível de lipídio $(2,51 \%$ do peso seco) e o um dos menores teores 
proteicos (5,60\% do peso seco). A Ceramium diaphanum (alga vermelha) obteve o maior teor proteico ( $14 \%$ do peso seco).

Mediante uma composição nutricional ampla, as algas podem ser aplicadas como suplementação proteica e mineral na alimentação animal, buscando um melhor desempenho zootécnico.

\subsection{Produção de algas}

Durante muito tempo a biomassa algal utilizada nos mais diversos usos era proveniente dos estoques naturais. No entanto, a colheita intensiva e desordenada levou a uma queda considerável das populações nativas, em particular aquelas de potencial econômico. No início da década de 1970 ficou claro que a maior parte das algas era retirada dos bancos naturais e que o cultivo era o melhor meio de aumentar a produção (NAYLOR, 1976).

Segundo dados da FAO (2013), referentes ao ano de 2011, a produção mundial de algas alcançou 21 milhões de toneladas de peso vivo, deste total $93 \%$ foi de aquicultura (Tabela 1 ). O mercado faturou um total de $U \$ 5,5$ bilhões de dólares, um valor menor àqueles apresentados no ano anterior, isto porque houve uma baixa na produção da Índia. Sendo $65 \%$ vindos das indústrias de coloides, $30 \%$ da indústria de alimentos e apenas $5 \%$ de outras indústrias.

Tabela 1. Produção mundial de macroalgas (milhões de toneladas)

\begin{tabular}{lccc}
\hline Continentes & $\mathbf{2 0 0 9}$ & $\mathbf{2 0 1 0}$ & $\mathbf{2 0 1 1}$ \\
\hline África & 0.11 & 0.14 & 0.14 \\
Américas & 0.09 & 0.01 & 0.02 \\
Ásia & 17.14 & 18.84 & 20.80 \\
Europa & 0.0 & 0.0 & 0.0 \\
Oceania & 0.01 & 0.01 & 0.01 \\
Total & 17.4 & 19.0 & 21.0 \\
\hline
\end{tabular}

Fonte: FAO, 2013. 
VASCONCELOS, B.M.F. e ARRUDA, A.M.V. Uso de gracilaria no desempenho de frango de corte. PUBVET, Londrina, V. 8, N. 20, Ed. 269, Art. 1797, Outubro, 2014.

A Ásia é o continente com maior produtividade de algas, principalmente devido a China e Japão, os maiores produtores mundiais de algas, o Brasil encontre-se entre os 20 principais produtores (FAO, 2013).

$\mathrm{Na}$ década de 70, a explotação descontrolada de agarófitas no nordeste brasileiro provocou uma diminuição drástica das reservas naturais, com o desaparecimento de váriosbancos ao longo da costa (CÂMARA-NETO, 1982). Nessa época, novos incentivos foram criados para realização de experimentos de cultivos no mar em alguns estados do litoral nordestino, sendo encontrados valores promissores de crescimento para algumas espécies de Gracilaria (LIMA et al., 1981). No entanto, não foram alcançadas produções em escala comercial.

Com incentivos fornecidos pela $F A O / O C B$, cultivos no mar utilizando espécies de Gracilaria em escala piloto foram realizados nos estados do Rio Grande do Norte, Ceará e Paraíba, sendo observado em alguns casos, elevados valores de crescimento, e hoje o cultivo foi consolidado nesta região (MARINHO-SORIANO, 2005; BEZERRA \& MARINHO-SORIANO, 2010). Cultivos de macroalgas foram também realizados no litoral baiano com o intuito de selecionar espécies que apresentassem maior potencialidade, sendo G. cornea escolhida como a mais viável para o sistema testado (ACCIOLY \& PAULA, 2002; ACCIOLY, 2005).

Atualmente, existem cultivos consolidados no Rio grande do Norte, Ceará e Paraíba. A macroalgas Gracilaria birdiae é a mais produzida, devido a sua facilidade de adaptação ao cultivo e ao grande conteúdo de ágar. As praias de Pitangui e Rio do Fogo no Rio Grande do Norte produzem a Gracilaria birdiae, em Icapuí (Ceará) há cultivo de Gracilaria birdiae, Gracilaria caudata e Gracilaria dominigensis (MARINHO-SORIANO et al., 2011).

$\mathrm{Na}$ região sul do Brasil o estado de Santa Catarina se destaca na maricultura, a Gracilaria domingensis e Kappaphycus alvarezii tem cultivos consolidados e em escala comercial (SALLES et al., 2010; HAYASHI et al. 2010). O Rio Grande do Sul e Paraná não possuem cultivos em larga escala, apenas em âmbito laboratorial (PELLIZZARI \& REIS, 2011). 
VASCONCELOS, B.M.F. e ARRUDA, A.M.V. Uso de gracilaria no desempenho de frango de corte. PUBVET, Londrina, V. 8, N. 20, Ed. 269, Art. 1797, Outubro, 2014.

A região sudeste, São Paulo e Rio de Janeiro implementaram o cultivo comercial da alga Kappaphycus alvarezii. Esta alga é cultivada como matériaprima para uma pequena indústria carragena (PELLIZZARI \& REIS, 2011).

Segundo relatório do Ministério de Pesca e Aquicultura, em 2008 somente quatro estados cultivavam macroalgas, e apenas dois gêneros eram produzidos a Gracilaria, no nordeste e a Kappaphycus, no sudeste. O cultivo da Kappaphycus é realizado apenas no litoral sudeste devido ao fato de ser uma espécie exótica, há todo um cuidado para não destruir a biodiversidade marinha brasileira (MPA, 2008).

No relatório foram recenseadas 74 unidades produtivas, das quais o Ceará representou $61 \%$ do total, seguido pelo Rio Grande do Norte com 20\%, Rio de Janeiro com $18 \%$ e Paraná com 1\%. Esta produção é comercializada em sua maior parte de forma seca, o que correspondeu a $59 \%$ do total, seguido do "pó" com $2 \%$ e "fresco" com 12\%. Outras formas de produto e os não informados somaram $10 \%$. No Rio Grande do Norte foi comercializado apenas alga em "pó", enquanto no Ceará observou-se outros produtos, tais como cosméticos e alimentos (MPA,2008).

De acordo com estes dados, o nordeste se destaca na produção de macroalgas, sendo o Ceará o maior produtor, o Rio Grande do Norte vem em segundo, porém a forma de comercialização dos cearenses agregam mis valor ao produto final, gerando uma renda maior.

\section{3 - GÊNERO Gracilaria}

Segundo a literatura demonstra as algas da divisão Rhodophyta apresentam um teor proteico elevado quando comparado às outras divisões de macroalgas. Sendo, portanto fortes candidatas a aplicação na alimentação animal como fonte de proteínas.

A Gracilaria é um gênero de alga marinha macroscópica da divisão Rhodophta, que pode apresentar talo cilíndrico ou achatado, filamentoso ou pseudoparenquimatoso, de comprimento que pode variar entre 0,1 a 5 metros, 
VASCONCELOS, B.M.F. e ARRUDA, A.M.V. Uso de gracilaria no desempenho de frango de corte. PUBVET, Londrina, V. 8, N. 20, Ed. 269, Art. 1797, Outubro, 2014.

de coloração entre algumas tonalidades de vermelho, podendo haver ocorrência de variantes da cor verde (PLASTINO et al., 1999).

Espécies representantes do gênero Gracilaria estão distribuídas no mundo todo, desde a linha do Equador até altas latitudes. Porém a maioria das espécies ocorre em águas tropicais e pode crescer entre a zona entre maré até o infralitoral raso. São comuns em locais protegidos do batimento de ondulações, suportam a exposição ao ar e tem alta tolerância as variações ambientais (SALLES, 2006).

As principais espécies encontradas na América do Sul são a Gracilaria chilensis na costa chinela e a Gracilaria gracilis na costa Argentina, ambas representantes de águas subtropicais. No Brasil, segundo Oliveira (1998), as principais representantes são a Gracilaria cornea, G. caudata, G. tenuifrons e G. birdie.

A G. birdie é uma alga extremamente cultivada no litoral nordestino, principalmente devido ao seu potencial de produção de ágar. O seu ciclo de vida é do tipo Polysihonia, apresentando uma fase haploide (gametófitos) e duas fases diploides (carposporófito e tetrasporófito) (COSTA \& PLASTINO, 2001) (Figura 1).

A G. birdiae possui poucos estudos com relação a sua composição nutricional e absorção dos nutrientes. Segundo Pires e colaboradores (2012) a composição química desta espécie demonstra um alto valor nutricional (Tabela 1), com elevado conteúdo proteico e de fibras, constituindo assim uma excelente fonte de nutrientes para humanos e animais.

Embora a quantidade de lipídios seja baixa, o perfil de ácido graxo das algas em geral é muito interessante, possuindo uma grande quantidade de ácidos graxos poliinsaturados, estes já são conhecidos como benéficos à saúde (VAN GINNEKEN et al., 2011). 
VASCONCELOS, B.M.F. e ARRUDA, A.M.V. Uso de gracilaria no desempenho de frango de corte. PUBVET, Londrina, V. 8, N. 20, Ed. 269, Art. 1797, Outubro, 2014.

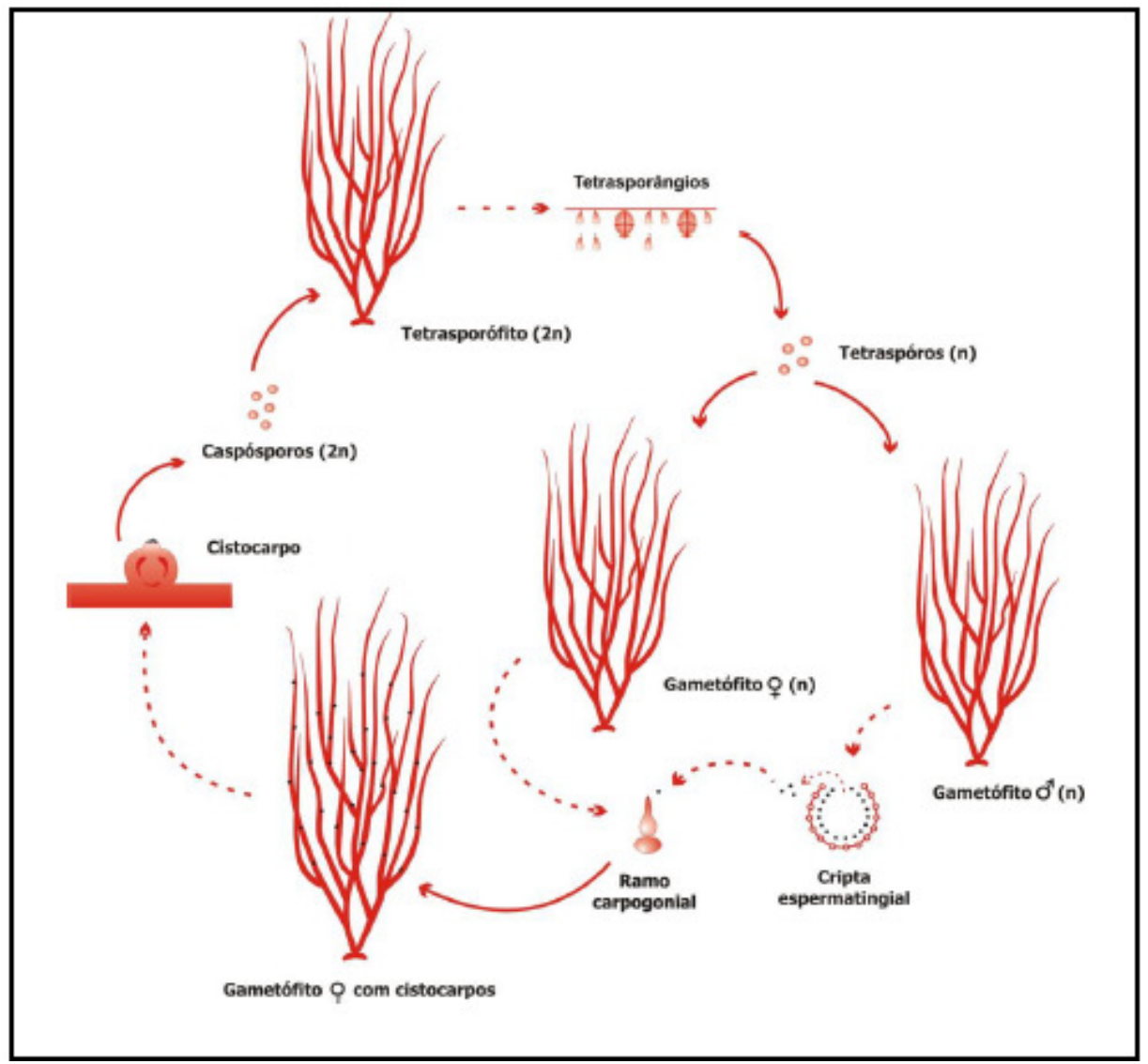

Figura 1. Ciclo de vida da Gracilaria birdiae. (Fonte: Carneiro, 2011).

Tabela 1 - Composição nutricional da Gracilaria birdiae.

\begin{tabular}{|cc|}
\hline Parâmetro & Valor \\
\hline pH & 6,58 \\
\hline Umidade (105\% $\mathbf{C})$ & 15,25 \\
\hline Proteínas (\%) & 12,62 \\
\hline Lipídios (\%) & 0,11 \\
\hline Cinzas (\%) & 5,58 \\
\hline Fibras Totais (\%) & 50,03 \\
\hline Fibras Insolúveis (\%) & 9,00 \\
\hline
\end{tabular}


VASCONCELOS, B.M.F. e ARRUDA, A.M.V. Uso de gracilaria no desempenho de frango de corte. PUBVET, Londrina, V. 8, N. 20, Ed. 269, Art. 1797, Outubro, 2014.

Diante dessas características, facilidade de cultivo e composição nutricional rica em proteínas, a G. birdiae, é uma excelente candidata para a suplementação animal, podendo melhorar o desempenho zootécnico dos frangos de corte, contudo estudos nesta área ainda são escassos e/ou inexistentes.

\section{4- USO DE ALGAS NA AVICULTURA}

Na alimentação animal a utilização de algas ainda é pouco explorada, sendo utilizada como uma alternativa para as dietas animais, para substituir ingredientes de custos elevados ou que apresentam riscos de escassez, como por exemplo, o fósforo e o calcário (PELÍCIA, 2007).

$\mathrm{Na}$ criação de frangos de corte, a alimentação representa a maior parcela dos custos de produção, podendo chegar até $70 \%$ do custo total. Em decorrência desse fato, bastante representativo, é que as empresas ligadas ao ramo de nutrição animal estão buscando novas fontes alternativas de alimentos, objetivando a redução nos custos de produção (CARLOS, 2011). De acordo com Damasceno et al. (2010) uma série de fatores foram fundamentais para que a avicultura de corte se apresente como uma das mais importantes fontes de proteína animal e dentre estes fatores, temos a aquisição de novos conhecimentos específicos na área de nutrição, genética, manejo e bem-estar das aves.

A pouca diversidade da microflora intestinal de aves recém-nascidas, é um dos fatores fundamentais a avicultura, além de ser considerada como um fator limitante para a digestão, também possibilita a colonização intestinal por patógenos entéricos. $O$ efeito negativo desse processo tem sido contornado, em parte, com o uso de promotores de crescimento (LORENÇON et al., 2007).

Os promotores de crescimento são os principais aditivos antimicrobianos de uso na alimentação animal, entre eles incluem-se os quimioterápicos e antibióticos. Estes são utilizados desde a década de 50 em doses subterapêuticas nas rações para melhorar o crescimento e a eficiência de produção, uma vez que impede que os microrganismos patogênicos invadam e 
VASCONCELOS, B.M.F. e ARRUDA, A.M.V. Uso de gracilaria no desempenho de frango de corte. PUBVET, Londrina, V. 8, N. 20, Ed. 269, Art. 1797, Outubro, 2014.

se multipliquem no intestino do animal, permitindo assim que os nutrientes da dieta sejam aproveitados para o tecido de crescimento do músculo ao invés do sistema imune. (BUTOLO, 1999; DIONIZIO, 2002; LODDI, 2003; CORNELI,2004; FLEMMING, 2005; LORENÇON et al., 2007).

Contudo, vários questionamentos têm sido feitos sobre o uso de antibióticos em rações para promover o desempenho de aves, em virtude da possível presença de resíduos em carne e ovos que possam alterar o balanço microbiano intestinal, além disso, o uso tem contribuído para o surgimento de resistência de bactérias patogênicas (MELO et al., 2010).

Portanto, nesta ótica, os prebióticos surgem como excelentes fontes alternativas. Estes produtos não são digeridos no intestino delgado, permitindo a fermentação e o fornecimento de substrato para uma determina colônia de bactérias benéficas que ao se desenvolver, garantirá o equilíbrio microbiano no epitélio intestinal (MELO et al., 2010).

As algas possuem um elevado teor de carboidratos não amiláceos, principalmente os oligossacarídeos, portanto elas são exímias candidatas a serem aplicadas como prebiótico na alimentação animal, porém as macroalgas ainda não foram aplicadas sob esta ótica, as pesquisas com macroalgas na avicultura tem se restringido a substituição de calcário calcítico pelo cálcio orgânico, vindo das macroalgas (BERTEAU \& MULLOY,2003).

A alga Lithothamnium calcareum é mais utilizada na avicultura, ela é uma Rhodophyta que apresenta uma grande quantidade de cálcio. Diversos estudos têm demonstrado seus efeitos no desempenho de animais, não só o frango.

Souza (2012) avaliaram a utilização de Lithothamnium calcareum como fonte de cálcio em galinhas poedeiras Dekalb White, e demonstraram que a inclusão de $1 \%$ desta alga na dieta das poedeiras melhoravam a qualidade e produção dos ovos, aumentando a espessura da casca e diminuindo a percentagem de ovos trincados.

Perali et al., (2003) referenciaram aumento na produção de ovos de cordonas japonesas (Coturnix japonica) em 4,16 pontos percentuais em 
VASCONCELOS, B.M.F. e ARRUDA, A.M.V. Uso de gracilaria no desempenho de frango de corte. PUBVET, Londrina, V. 8, N. 20, Ed. 269, Art. 1797, Outubro, 2014.

relação à testemunha na adição de $0,25 \%$ deste produto. Porém o aumento na produção de ovos não foi observado por Melo et al. (2008a; 2008b), os quais avaliaram a utilização da farinha de algas calcáreas Lithothamnium calcareum no desempenho e qualidade de ovos de codornas japonesas, e observaram que o suplemento mostrou evidencias de melhoria na casca dos ovos e um aumento significativo no peso da gema, porém as características de desempenho não foram influenciadas pela utilização da farinha de algas calcáreas.

Efeitos de melhora na conversão alimentar foram observados por Pope et al. (2002), em frangos de corte suplementados com Lithothamnium calcareum, os quais apresentaram maior ganho de peso e melhor rendimento de peito, devido a melhora na conversão alimentar.

Sobre características de carcaça de frangos, não foram verificados efeito significativo do uso de farinha de algas calcáreas sobre a composição da carcaça de frangos de corte (ZANINI et al., 2002).

Carlos et al. (2011) avaliaram o uso da Lithothamnium calcareum em frangos de corte. Os autores adicionaram a alga para aumentar a quantidade de cálcio na fase inicial (1-21 dias) e na fase de crescimento (21-42 dias) e analisaram nas duas fases e no período total. Os resultados obtidos demonstraram que na fase inicial não houve ganho de peso e na fase de crescimento e período total não houve alterações significativas, demonstrando que o uso pode ser introduzido, mediante análise de viabilidade econômica.

Cedro e colaboradores (2011) utilizaram algas marinhas e óleo de peixe para enriquecerem ovos de galinha com ômega 3. Eles adicionaram uma suplementação de $1,5 \%$ dos componentes da dieta com farinha de algas e $1,8 \%$ com óleo de peixe, posteriormente avaliaram a composição nutricional do ovo e determinaram o perfil lipídico demonstrando um enriquecimento de ômega 3 nos ovos postos pelas poedeiras alimentadas com algas e óleo de peixe.

O uso de algas na avicultura ainda é muito relacionado as galinhas poedeiras, sendo os frangos de corte um pouco esquecidos, contudo 
VASCONCELOS, B.M.F. e ARRUDA, A.M.V. Uso de gracilaria no desempenho de frango de corte. PUBVET, Londrina, V. 8, N. 20, Ed. 269, Art. 1797, Outubro, 2014.

atualmente trabalhos estão sendo desenvolvidos nesta área, buscando tipos de algas que melhorem o desempenho zootécnico, como a qualidade da carcaça e tenho um aumento na digestibilidade, fazendo com que o animal converta melhor os nutrientes.

\section{5- CONCLUSÕES}

As algas apresentam um grande potencial para a avicultura, principalmente devido às características nutricionais e os perfis de ácidos graxos. A alga Lithothamnium calcareum é uma das mais utilizadas, principalmente como fonte de cálcio para poedeiras, o uso desta alga para frango de corte tem se mostrado benéfico.

Contudo, a inexistência de cultivos destas algas a tornam ineficazes para a produção comercial. A Gracilaria birdiae é uma alga da mesma divisão da L.calcareum e possui uma excelente composição nutricional, rica em proteínas, desmontando que pode ser aplicada como fonte proteica para frangos de corte, melhorando a carcaça e o desempenho zootécnico.

\section{6-REFERÊNCIAS BIBLIOGRÁFICAS}

ACCIOLY, M.C. Esforços para estabelecer cultivos artesanais de macroalgas marinhas na Bahia. In: Anais da X Reunião Brasileira de Ficologia. Salvador 2004. Rio de Janeiro, Museu Nacional. Série Livros 10. pp. 107-114, 2005.

ACCIOLY, M.C. \& PAULA, E.J. 2002. Seleção de rodofíceas com potencial econômico para cultivo na região do baixo-sul baiano. In: Anais da IX Reunião Brasileira de Ficologia. Santa Cruz, Aracruz, Espírito Santo. Sociedade Brasileira de Ficologia \& Fundação Ecossistemas do Espírito Santo. pp. 107, 2002.

ALGAS VERDES (2012). Algas verdes: o que caracteriza? Disponível em: <http://www.dbi.uem.br/algas-verdes.pdf. >. Acesso em: 29 out. 2013.

BERTEAU, O.; MULLOY, B. Sulfated fucans, fresh perpectives: structures,functions, and biological properties of sulfated fucans and overview of enzymes active toward this class of polysaccharide. Glycobiology, v.13, n.6, p.29-40, 2003.

BUTOLO, J. E. Uso de biomassa de levedura em alimentação animal: propriedades, custo relativo a outras formas de nutrientes. IN: Produção de Biomassa de Leveduras: utilização em alimentação humana e animal. Campinas. Campinas: ITAL; p.70, 1996. 
CÂMARA-NETO C. Cultivo de agarófitas. Uma perspectiva econômica para o litoral do Nordeste. Série Ciência \& Tecnologia 23p, 1982.

CALADO, C. M. B.; PIRES, V. C. F.; SANTOS, K. M. de A. Dos.; AlbuQUeRQue, A. P.; FLORENTINO, E. R. Algas comestíveis: comparação nutricional entre espécies de gracilaria (G. cornea e G. domingensis) de ocorrências no litoral nordestino. In: ENCONTRO NACIONAL DE EDUCAÇÃO, CIÊNCIA E TECNOLOGIA, 1., 2011, Campina Grande.Trabalho. Campina Grande: Realize, 2011. p. 20 - 28.

CARneiro, J. G.; CAVAlCANTE, A. B. D.; TEIXeirA, D. M. A.; PEReirA, I. K. D.; CARneiro, M.A.; BARBOSA, M.C.F. Potencial nutricional das algas vermelhas Hypnea musciformis e Solieria filiformis: Análises da composição centesimal. In: CONGRESSO NORTE-NORDESTE DE PESQUISA E INOVAÇÃO, 7., 2012, Palmas.Trabalho de Congresso. Palmas: Propi, 2012. p. 1 - $5 . \quad$ Disponível em: <http://propi.ifto.edu.br/ocs/index.php/connepi/vii/paper/viewFile/1227/1765>. Acesso em: 23 ago. 2013.

CARNEIRO, M. A. A. Fenologia, aspectos ecofisiológicos e seleção de linhagens em cultivos no mar de Gracilaria birdiae. Tese de doutorado. Universidade de São Paulo. São Paulo-SP, p. 50, 2011.

CEDRO, T. M. M.; CALIXTO, L. F. L.; GASPAR, A. Proporções entre ácidos graxos poliinsaturados em ovos comerciais convencionais e enriquecidos com ômega-3. Ciênc. Rur., Santa Maria, v. 41, n. 4, p.706-711, abr. 2011.

CORNELI, J. Avaliação de promotores de crescimento alternativos em substituição aos convencionais sobre o desempenho, característica de carcaça e morfologia intestinal em frangos de corte. Dissertação (Mestrado em Zootecnia) - Universidade Federal de Santa Maria, Rio Grande do Sul, 2004, 59 f.

COSTA NETO, J. M.; TEXEIRA, R. G.; SÁ, M. J. C.; LIMA, A. E.; JACINTO-ARAGÃO, G. S.; TEXEIRA, M. W.; FILHO, E. F. M.; TORÍBIO, J. M. M. L.; AZEVEDO, A. S. Farinha de algas marinhas (Lithothamnium calcareum) como suplemento mineral na cicatrização óssea de auto enxerto cortical de cães. Revista Brasileira Saúde Produção Animal, Salvador, v.11, n. 1, p. $217-230,2010$.

COSTA V. L. \& PLASTINO, E. M. Histórico de via de espécimes selvagens e variantes cromáticas de Gracilaria sp. Revista Brasileira de Botânica 24: 483-490, 2001.

DARCY-VRILLON, B. Nutritional aspects of the developing use of marine macroalgae for the human food industry. International Journal of Food Science and Nutrition, 44, p. 23-35. 1993.

DAWCZYNSKI, C.; SCHUBERT, R.; JAHREIS, G. Amino acids, fatty acids, and dietary fibre in edible seaweed products. Food Chem 103: 891-899. 2007.

DIONIZIO, M. A.; BERTECHINI, A. G.; KATO, R. K.; TEIXEIRA, A. S. Prebióticos como promotores de crescimento para frangos de corte - desempenho e rendimento de carcaça. Ciência Agrotécnica, Lavras. p. 1580-1587, dez., 2002.

FAO: Fisheries and Aquaculture Department . 2013. Global Aquaculture Production Statistics for the year 2011. Disponível em: ftp://ftp.fao.org/FI/news/GlobalAquacultureProductionStatistics2011.pdf. Acesso 21 nov 2013. 
FLEMMING, J. S. Utilização de leveduras, probióticos e mananoligossacarídeos (MOS) na alimentação de frangos de corte. 2005. 109 f. Tese (Doutorado em Tecnologia de Alimentos) - Universidade Federal do Paraná, Paraná, 2005.

FRIKHA, F; KAMMOUN, M; HAMMAMI, N.; MCHIRGUI, R.A; BELBAHRI, L; GARGOURI, Y; MILED, N.; BEN-REBAH, F. Chemical composition and some biological activities of marine algae collected in Tunisia. Cienc. Mar, Ensenada, v. 32, n. 2, p.113-124, 2011.

GOETZ, P. Phytothérapie de I'ostéoporose. Phytothérapie, Uberlândia, v.6, n.1, p.33-34, 2008.

HAYASHI L, SANTOS AA, FARIA GSM, NUNES BG, SOUZA MS, FONSECA ALD, BARRETO PLM, OLIVEIRA EC, BOUZON ZL. Kappaphycus alvarezii (Rhodophyta, Areschougiaceae) cultivadas em águas subtropicais do sul do Brasil. J Appl Phycol . 2010.

LODDI, M. M. Probióticos, prebióticos e acidificantes orgânicos em dietas para frangos de corte, 2003. 52 f. Tese (Doutorado em Zootecnia) - Faculdade de Ciências Agrárias e Veterinárias, Universidade Estadual Paulista "Júlio de Mesquita Filho", Jaboticabal, 2003.

LORENÇON, L.; NUNES, R. V.; POZZA, P. C.; POZZA, M. S. S.; APPELT, M. D.; SILVA, W. T. M. Utilização de promotores de crescimento para frangos de corte em rações fareladas e peletizadas. Acta Scientiarum. Animal Science, v. 29, n. 2, p. 151-158, 2007.

MABEAU, S. FLEURENCE, J. Seaweed in food products:Biochemical and nutritional aspects. Trends in Food Science and Technology. 4, 103-107, 1993.

MACHADO, D. A. V. et al. Levedura (Sacharomyces cerevisiae) spray-dry, autolisada e parede celular de levedura na alimentação de frango de corte. Vet. Zootec. 17(4): 541-551, 2010.

MARINHO-SORIANO, E.; PINTO, E.; YOKOYA, N. S.; COLEPICOLO, P.; TEXEIRA, V. L.; YONESHIGUE-VALENTIN, Y. . Frontiers on algae bioactive compounds. Brazilian Journal Of Pharmacognosy, Rio de Janeiro, n. , p.1-1, abr. 2011.

MARINHO-SORIANO, E. Cultivo experimental de Gracilaria no Rio Grande do Norte. pp. 115124. In: Anais da X Reunião Brasileira de Ficologia. Salvador 2004. Rio de Janeiro, Museu Nacional. Série Livros 10, 2005.

MARINHO-SORIANO, E.; PINTO, E.; YOKOYA, N. S.; COLEPICOLO, P.; TEXEIRA, V. L.; YONESHIGUE-VALENTIN, Y. . Frontiers on algae bioactive compounds. Brazilian Journal Of Pharmacognosy, Rio de Janeiro, n. , p.1-1, abr. 2011.

MAURÍCIO A.; JACINTO, L.; SERÔDIO, L.; CLEMENTE, M. As algas na nossa alimentação. Ciências da natureza. p.1-10, 2011.

McHUGH, D.J. A guide to the seaweed industry. Rome: Food and Agriculture Organization of The United Nations, FAO Fisheries Technical Paper 441, 111 p., 2003.

MELO, T.V.; MENDONÇA, P.P.; MOURA, A.M.A.; LOMBARDI, C.T.; FERREIRA, R.A.; NERY, V.L.H. Solubilidad in vitro de algunas fuentes de cálcio utilizadas em alimentacion animal. Archivos de zootecnia, Cordoba, v.55, n 211, p. 297-300, 2006.

MELO, T.V., R.A. FERREIRA, V.C. OLIVEIRA, J.B.A. CARNEIRO, A.M.A. MOURA, C.S. SILVA E NERY, V.L.H.. Calidad del huevo de codornices utilizando harina de algas marinas y fosfato monoamónico. Arch. Zootec., 57: 313-319, 2008a. 
MELO, T.V., R.A. FERREIRA, J.B.A. CARNEIRO, V.C. OLIVEIRA, A.M.A. MOURA, C.S. SILVA E V.L.H. NERY. Rendimiento de codornices japonesas utilizando harina de algas marinas y fosfato monoamónico. Arch. Zootec., 57: 381-384, 2008b.

MELO, T. de S.; SILVA, J. H. V. da; RIBEIRO, M. L. G.; TEIXEIRA, E. N. M; SILVA, C. T.; LACERDA, P. B. Anais. In: 47A REUNIÃO ANUAL DA SOCIEDADE BRASILEIRA DE ZOOTECNIA, 47., 2010, Salvador. Uso de Prebiótico na Ração de Frangos de Corte em Fase PréInicial. Salvador: Sbz, 2010. p. 10 - 15.

MENDEZ, L. T. Guia de Biodiversidad: Macrofaunas y Algas Marinas. CREA, v.1, no 4 2002.

MPA - Ministério de Pesca e Aquicultura (2008). Censo Aquícola Nacional: Ano 2008. Disponível

http://www.mpa.gov.br/images/Docs/Informacoes_e_Estatisticas/Censo_maio2013-2.pdf. Acesso em 23 nov. 2013.

NAYLOR, J. Production, Trade and Utilization of Seaweeds and Seaweed Products. FAO Fisheries Technical Paper. Rome: Food and Agriculture Organization, n. 159, 1976.

PATARRA, A.R.F. Pesquisa de Ácidos Gordos em macroalgas marinhas do litoral dos Açores. Dissertação de mestrado. Universidade do Porto, 2008.

PELÍCIA, K. Alternative Calcium Source Effects on Commercial Egg Production and Quality. Brazilian Journal of Poultry Science, v.9 / n.2 / 105 - 109, Apr - Jun, 2007.

PELLIZZARI, F. \& REIS, R.P. Seaweed cultivation on the Southern and Southeastern Brazilian Coast. Revista brasileira de farmacognosia 21 (2): 305-312, 2011.

PERALI, C., M. ARANOVICH, M.W. SANTOS, S. ARANOVICH, D.M.F. COSTA, G.M. SILVA E ROCHA, V.F. Efeito de diferentes níveis de adição do Suminal ${ }^{\circledR}$ sobre a produção e peso de ovos de codornas alimentadas com concentrados. 40 Reunião Anual da Sociedade Brasileira de Zootecnia. Santa Maria, Anais (CD) .... Sociedade Brasileira de Zootecnia. Santa Maria, 2003.

PIRES, V. C. F.; CALADO, C. M. B.; SANTOS, K. M. de A. Dos.; AlBUQUERQUE, A. P.; FLORENTINO, E. R. Caracterização físico-química da macroalga Gracilaria domingensis. In: CONGRESSO BRASILEIRO DE QUÍMICA, 52., 2012, Recife. Trabalho. Rio de Janeiro: Associação Brasileira de Quimica, 2012. v. 1, p. 1 - 1 . Disponível em: <http://www.abq.org.br/cbq/2012/trabalhos/10/1493-13804.html>. Acesso em: 14 out. 2013.

POPE, H.R., C.M. OWENS, L.C. CAVITT, J.L. EMMERT E S.J. TAYLOR. Efficacy of marigro in supporting growth, carcass yield and meat quality of broilers. 91st Annual Meeting Abstracts. The Southern Poultry Science Society. Poscal 80 (Suppl. 1). p. 25, 2002.

RAVEN, P. H.; EVERT, R. F.; EICHHORN, S. E. (1996). Algas verdes, vermelhas e pardas. Disponível em: http://www.cienciasbiologicas.hpg.ig.com.br/algasciano.htm. Acesso em 27 set 2013.

RUPÉREZ, Pilar; GÓMEZ-ORDÓÑEZ, Eva; JIMÉNEZ-ESCRIG, Antonio. Dietary fibre and physicochemical properties of several edible seaweeds from the northwestern Spanish coast. Food Research International, Estados Unidos, n. , p.2289-2294, 2010. 
SALLES JP, SCHERNER F, FANGANIELLO M, BOUZON ZL, HORTA PA Cultivo de algas nativas Gracilaria domingensis(Rhodophyta) no Sul do Brasil. Braz Arc Biol Tecno 53 : 633640, 2010.

SANTOS, F. L.; LIMA-ARAÚJO, K. G. de; ROCHA-LEÃO, M. H. M. da.; SANTOS, B. N. C; ANDRADE, C. T. Composição centesimal da alga Kappaphycus alvarezii. In: CONGRESSO BRASILEIRO DE FICOLOGIA, 14., 2012, João Pessoa. Trabalho de Congresso. João Pessoa: SBFIC, 2012. p. 1 - 5.

TABARSA, M. REZAEI, M. RAMEZANPOUR, Z. ROBERTWAALAND. J. Chemical compositions of the marine algae Gracilaria salicornia (Rhodophyta) and Ulva lactuca (Chlorophyta) as a potential food source. Journal Sciense Food Agriculture, 2012.

VAN GINNEKEN, V. J.T.; HELSPER, J.P.F.G.; VISSER, W. DE; KEULEN, H. VAN; BRANDENBURG, W.A. Polyunsaturated fatty acids in various macroalgal species from north Atlantic and tropical seas. Lipids In Health And Disease, Estados Unidos, n. , p.1-8, set. 2011. Disponível em: http://www.lipidworld.com/content/10/1/104>. Acesso em: 23 set. 2013.

ZANINI, S.F., M.A.G. CARVALHO, G. COLNAGO, R. QUILULA, A. M. N. KORRES, K. S. SANTOS, C. O. SILVA JÚNIOR, A.C. SOARES E F. FAVA. 2002. Composição da carcaça de frangos de corte submetidos a dieta com farinha de algas. Rev. Centro Univ. Vila Velha (ES), 3 (janeiro/julho): 45.

ZYELBERSTJN, D.; GRAÇA, C. T. O agronegócio das carnes: mudanças nos mercados, tecnológicos e organizacionais. IN: Seminário Degussa sobre Nutrição de Aves. São Paulo: Degussa, p. 1-9, 2002. 